\title{
Effect of Anagliptin versus Sitagliptin on Inflammatory Markers: Sub-Analysis from the REASON Trial
}

This article was published in the following Dove Press journal: Diabetes, Metabolic Syndrome and Obesity: Targets and Therapy

\author{
Hiroki Teragawa (D) \\ Takeshi Morimoto (D) ${ }^{2}$ \\ Yuichi Fujii' \\ Tomohiro Ueda' \\ Mio Sakuma ${ }^{2}$ \\ Michio Shimabukuro ${ }^{3}$ \\ Osamu Arasaki ${ }^{4}$ \\ Koichi Node ${ }^{5}$ \\ Takashi Nomiyama ${ }^{6}$ \\ Shinichiro Ueda ${ }^{7}$ \\ 'Department of Cardiovascular Medicine, \\ JR Hiroshima Hospital, Hiroshima, Japan; \\ ${ }^{2}$ Department of Clinical Epidemiology, \\ Hyogo College of Medicine, Nishinomiya, \\ Japan; ${ }^{3}$ Department of Diabetes, \\ Endocrinology and Metabolism, Fukushima \\ Medical University, Fukushima, Japan; \\ ${ }^{4}$ Department of Cardiology, Tomishiro \\ Central Hospital, Tomigusuku, Okinawa, \\ Japan; ${ }^{5}$ Department of Cardiovascular \\ Medicine, Saga University, Saga, Japan; \\ ${ }^{6}$ Department of Endocrinology and \\ Diabetes Mellitus, Fukuoka University, \\ Fukuoka, Japan; ${ }^{7}$ Department of Clinical \\ Pharmacology and Therapeutics, University \\ of the Ryukyus, Nishihara, Okinawa, Japan
}

Correspondence: Hiroki Teragawa Department of Cardiovascular Medicine, JR Hiroshima Hospital, 3-I-36

Futabanosato, Higashi-ku, Hiroshima 7320052, Japan

Tel +8I-82-262-|| $|7|$

Fax $+81-82-262-1499$

Email hiroteraga7I@gmail.om
Purpose: Experimental evidence has suggested that dipeptidyl peptidase-4 (DPP-4) inhibitors have an anti-inflammatory effect as well as a glucose-lowering effect, but this has yet to be confirmed in diabetic patients. Therefore, we examined the anti-inflammatory effects of two kinds of DPP-4 inhibitors in patients who participated in the randomized evaluation of anagliptin (ANA) vs sitagliptin (SITA) on low-density lipoprotein cholesterol in diabetes (REASON) Trial, which compared low-density lipoprotein-cholesterol lowering effects between (ANA) and SITA in patients with type 2 diabetes, dyslipidemia, and atherosclerotic vascular lesions.

Patients and Methods: The studied patients consisted of 177 patients who received ANA $200 \mathrm{mg}$ per day and 176 patients who received SITA $50 \mathrm{mg}$ per day for 52 weeks. We measured high-sensitivity C-reactive protein (hs-CRP), white blood cells (WBC), and interleukin-6 (IL-6) before and after treatment for 52 weeks, and the changes in inflammatory markers were measured as the differences between baseline and 52 weeks. Furthermore, we checked the relationship between the change in hs-CRP and several clinical factors such as the baseline hs-CRP level, use of a moderate-intensity statin, presence of coronary artery disease (CAD) and taking a previous DDP-4 inhibitor.

Results: The levels of the inflammatory markers hs-CRP, WBC, and IL-6 were determined to have not significantly changed from baseline to the final follow-up in each arm; furthermore, the changes in these markers were not significantly different between the two groups. The change in hs-CRP level was not affected by the baseline hs-CRP level, use of a moderate-intensity statin, presence of coronary artery disease, and absence of prior DPP-4 inhibitor use.

Conclusion: In this sub-analysis from the REASON Trial, taking a DPP-4 inhibitor, either ANA or SITA, for 52 weeks did not affect the levels of inflammatory markers.

Keywords: C-reactive protein, dipeptidyl peptidase-4, DPP-4 inhibitor, inflammation, interleukin-6, white blood cell

\section{Introduction}

Diabetes mellitus (DM) has been identified as one of the strongest risk factors for atherosclerotic disease. ${ }^{1,2}$ In Europe and the United States, myocardial infarction is the direct cause of death in $40-50 \%$ of patients with diabetes, and the number of patients with diabetes that die as a direct result of ischemic heart disease is reportedly increasing. In Japan, the number of patients with DM has seen a significant increase over the past few decades. ${ }^{3}$ Thus, a multifactorial intervention that includes control of blood 
glucose, blood pressure, and lipid levels is crucial for the prevention of DM-related vascular disease. ${ }^{2,4,5}$ Regarding drug treatment, dipeptidyl peptidase-4 (DPP-4) inhibitors were determined to enhance the action of glucagon-like peptide-1 (GLP-1), identified as the most physiologically important incretin, and they consequently show various actions, such as the stimulation of glucose-dependent insulin secretion, inhibition of glucagon secretion and gastric emptying, and regulation of appetite. ${ }^{6}$ DPP-4 inhibitors have gained widespread use in the clinical setting because they have been reported to improve hemoglobin A1c levels with less frequent severe complications, although favorable effects on cardiovascular outcomes from adding DPP-4 inhibitors to usual care in diabetic patients with established cardiovascular diseases have yet to be established. ${ }^{7-10}$

It has been reported that inflammation is closely related to the development of atherosclerosis, ${ }^{11}$ and that an improvement of the inflammatory response may reduce the onset of cardiovascular events. ${ }^{12,13}$ Thus, much interest has been focused on the anti-inflammatory effect of drugs, including antidiabetic drugs such as DPP-4 inhibitors, that are used to treat patients with cardiovascular risk factors or established cardiovascular diseases. ${ }^{14}$

The Randomized Evaluation of Anagliptin vs Sitagliptin On low-density lipoproteiN cholesterol in diabetes (REASON) Trial has recently compared the effectiveness of anagliptin (ANA) and sitagliptin (SITA) in reducing lowdensity lipoprotein cholesterol (LDL-C) in patients with type 2 diabetes, dyslipidemia, and existing atherosclerotic vascular lesions. The study demonstrated that ANA was superior to SITA in lowering LDL-C by an estimated treatment difference of $4.5 \mathrm{mg} / \mathrm{dL}{ }^{15}$ This finding might cause some difference in anti-inflammatory effects with ANA and SITA, because it has been shown that a reduction in LDL-C can lower the inflammatory response. ${ }^{16}$ Thus, in this present sub-analysis of the REASON Trial, we determined whether the two different DPP-4 inhibitors, ANA and SITA, exhibit anti-inflammatory effects.

\section{Patients and Methods}

\section{Trial Design and Participants}

The design and participants of the REASON Trial were previously reported in the protocol paper. ${ }^{17}$ In brief, the REASON Trial was a multicenter, randomized, open-label, active-controlled, parallel-group trial that compared the effectiveness of ANA versus SITA in reducing LDL-C in patients with type 2 diabetes, dyslipidemia, and existing atherosclerotic vascular lesions. Eligible participants were adults (age $\geq 20$ years) diagnosed with type 2 diabetes that was treated with diet and exercise alone or in combination with hypoglycemic agents who had existing atherosclerotic vascular lesions, were treated with statins for dyslipidemia for $\geq 8$ weeks, and had documented LDL-C $\geq 100$ $\mathrm{mg} / \mathrm{dL}$ in at least one measurement after using statins. Key exclusion criteria were as follows: type 1 diabetes; triglycerides $\geq 400 \mathrm{mg} / \mathrm{dL}$ in a past fasting blood sample; pregnancy, potential pregnancy, or breastfeeding in women; severe infections; surgery; serious trauma; serum creatinine level $\geq 2.4 \mathrm{mg} / \mathrm{dL}$ for men or $\geq 2.0 \mathrm{mg} / \mathrm{dL}$ for women; and use of GLP-1 receptor agonists. In addition, patients who had regularly taken anti-inflammatory drugs, such as nonsteroidal anti-inflammatory drugs and steroids, were excluded from this present analysis.

\section{Randomization and Intervention}

The randomization and interventions were also reported in the previous paper. ${ }^{17}$ In brief, participants in the ANA group were given ANA $100 \mathrm{mg}$ orally twice daily for 52 weeks. If the effects were deemed insufficient, the dose could be increased to $200 \mathrm{mg}$ orally twice daily. Meanwhile, participants in the SITA group were given SITA $50 \mathrm{mg}$ orally once daily for 52 weeks. If the effects were deemed insufficient, the dose could be increased to $100 \mathrm{mg}$ per day. If the participants used antidiabetic drugs other than DPP-4 inhibitors at the start of the trial, the study drug was administered concomitantly, and such antidiabetic drugs were not replaced. The treatment assignment was not concealed from the participants or the treating physicians.

During the trial period, hypoglycemic agents and antidyslipidemia drugs (statins, ezetimibe, anion-exchange resin agents, fibrates, and eicosapentaenoic acid) were not added, and their dosages were not changed; a change in the dose of insulin was not considered a change in hypoglycemic agent. The need for other therapy was determined by the physician in charge; however, changes in other medications with possible effects on the glycemic and lipid control were deemed prohibited. Clinical research coordinators regularly monitored the participants and their physicians in order to ensure adherence to the study medication/dose at every visit. If a violation was found, then the participants were removed as per protocol.

\section{Measurements}

The blood samples were analyzed at the core laboratory (SRL Inc., Tokyo, Japan). Levels of the inflammatory 
markers high-sensitivity C-reactive protein (hs-CRP, mg/ L), white blood cells (WBC/ $/ \mathrm{mm}^{3}$ ), and interleukin-6 (IL-6, $\mathrm{ng} / \mathrm{mL}$ ) were examined at baseline and the final follow-up. In this present sub-analysis, the differences in these inflammatory markers were expressed as the marker levels at final follow-up minus those at baseline. To check the relationship between the difference in hs-CRP and clinical parameters, the effects of the baseline level of hs-CRP, the use of moderate-intensity statins, the presence of established coronary artery disease (CAD), and the absence of previous DPP-4 inhibitor use were then evaluated.

\section{Definitions}

Regarding the statins taken, a moderate-intensity statin was defined as taking atorvastatin $(10-20 \mathrm{mg})$, rosuvastatin $(5-10 \mathrm{mg})$, simvastatin $(20-40 \mathrm{mg})$, or pravastatin (40$80 \mathrm{mg}$ ); meanwhile, a mild-intensity statin was defined as taking these statins at less than the doses listed. ${ }^{18}$ Established CAD was defined as a previous medical history of acute coronary syndrome, history of percutaneous coronary intervention or coronary artery bypass graft, or significant coronary stenosis $\geq 75 \%$ on coronary angiography or coronary computed tomography accompanied by ischemic chest symptoms. Hypertension was defined as the presence of systolic blood pressure $\geq 135 \mathrm{~mm} \mathrm{Hg}$ and/or diastolic blood pressure $\geq 85 \mathrm{~mm} \mathrm{Hg}$ or the use of antihypertensive drugs.

\section{Statistical Analyses}

All analyses were performed under the intention-to-treat principle. Categorical variables were expressed as frequencies with percentages, whereas continuous variables were expressed as means with standard deviations or medians with interquartile ranges. The subgroup classification of hs-CRP was based on the median value. All statistical analyses were performed at the data center (Institute for Clinical Effectiveness) by the study statistician (T. Morimoto), who used JMP 13.1 (SAS Institute Inc., Cary, NC) and SAS 9.4 (SAS Institute Inc., Cary, NC) based on the statistical analysis plan. All $P$-values were determined to be two-sided, and $P<0.05$ was considered significant.

\section{Results}

\section{Patient Characteristics}

Among the 353 participants, 177 patients were grouped into the ANA group and 176 patients into the SITA group
(Figure 1). The patient characteristics are summarized in Table 1 . The presence of past medical history, including established CAD; use of medications, including aspirin; and levels of inflammatory markers at baseline were determined to be not significantly different between the two groups.

\section{The Changes in the Inflammatory Markers}

There were no significant differences between the two groups in terms of the hs-CRP, WBC, and IL-6 levels at baseline (Table 1). The changes in these inflammatory markers from baseline to the final follow-up in the two groups are presented in Table 2. No significant differences from baseline to the final follow-up were noted in either arm, and the changes in these markers were determined to not significantly differ between the two groups. Because there were no significant differences in these markers, we performed the next sub-analysis using only hs-CRP.

\section{Relationship Between the Change in hs- CRP and Clinical Parameters}

First, the change in hs-CRP was deemed to not be associated with the changes in LDL-C or hemoglobin A1c in the REASON Trial. To check the factors responsible for the change in hs-CRP in the two groups, the hs-CRP level at baseline (median value $0.658 \mathrm{mg} / \mathrm{L}$ ), use of moderateintensity statins, presence of established CAD, and absence of prior DPP-4 inhibitor use were evaluated. The change in hs-CRP in the two groups was not affected by these factors (Figure 2).

\section{Discussion}

In this sub-analysis of the REASON Trial, we determined whether the effects of ANA and SITA on inflammatory markers were different in diabetic patients with high cardiovascular burden. Our findings showed no significant differences in the inflammatory markers hs-CRP, WBC, and IL- 6 after the use of these DPP-4 inhibitors in such patients. In addition, the difference in hs-CRP in the two groups was not affected by the hs-CRP value at baseline, use of moderate-intensity statins, presence of established CAD, or prior use of DPP-4 inhibitors.

DM has been identified as a major risk factor for the development of atherosclerotic disease, ${ }^{2}$ and atherosclerosis is considered to be an inflammatory disease. ${ }^{11}$ Thus, much interest has focused on the anti-inflammatory effect 


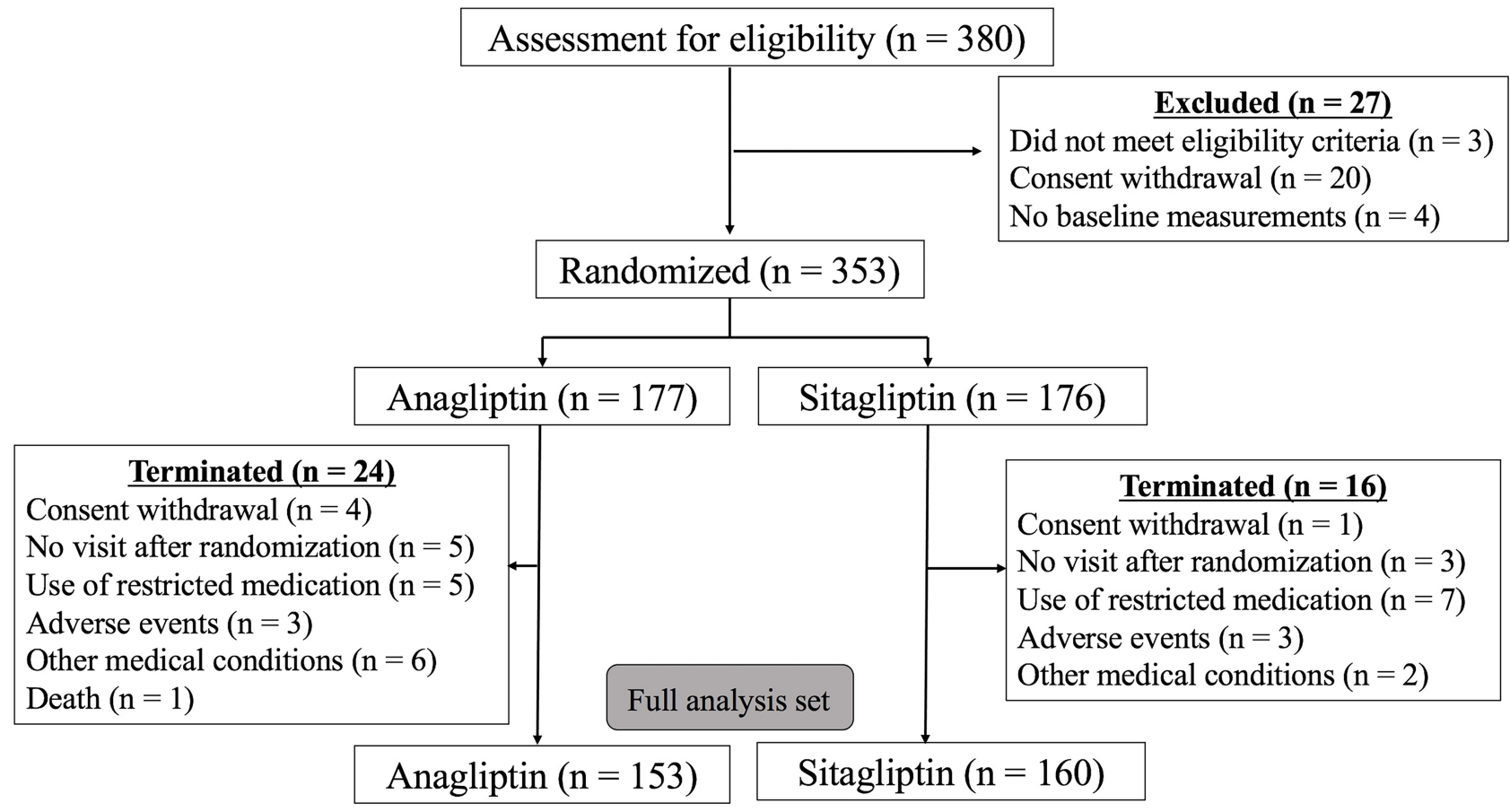

Figure I Participants' flowchart.

of antidiabetic drugs. Actually, there have been several studies showing the presence of an anti-inflammatory effect of antidiabetic drugs, including thiazolidinediones, ${ }^{19,20}$ GLP-1 receptor agonists, ${ }^{21,22}$ and sodium glucose co-transporter 2 inhibitors. ${ }^{23}$ In the REASON Trial, we used two different DPP-4 inhibitors, and there have been some studies focusing on the anti-inflammatory effects of DPP-4 inhibitors. ${ }^{14,24-29}$

In terms of the relationship between DPP-4 and inflammation, some experimental studies have shown that DPP-4 is expressed on macrophages, especially in visceral adipose tissue, and is involved in $\mathrm{T}$ cell proliferation and activation. ${ }^{14,30}$ Furthermore, it has been shown that tumor necrosis factor-alpha can cause the release of soluble DPP- $4,{ }^{14,31}$ which acts as an agonist for proteaseactivated receptor 2 on vascular cells, an activator of downstream cyclooxygenase, and a promotor of endothelial release of the vasoconstrictor thromboxane A2. ${ }^{14,32}$ In addition, it has been shown that this type of soluble DPP-4 in a concentration-dependent manner can activate extracellular signal-regulated kinase $1 / 2$, induce phosphorylation of the nuclear factor kappa B subunit p65, and increase inducible nitric oxide synthase activity and expression and secretion of proinflammatory cytokines. ${ }^{14}$ Thus, these experimental results demonstrate the presence of a relationship between DPP-4 and inflammation and further show the possibility that both soluble- and membrane-bound DPP-4 contain a catalytic site that can be inhibited by DPP-4 inhibitors. ${ }^{14}$

Regarding clinical studies, there have been several studies investigating the anti-inflammatory effects of DPP-4 inhibitors, especially SITA and ANA, which were utilized in the REASON Trial. ${ }^{24-29}$ In the studies using SITA, some showed a significant reduction in the level of CRP ${ }^{24,26}$ but others showed no significant changes in CRP levels. ${ }^{25,28}$ In one study using ANA, the level of CRP was reduced at 12 weeks but not at 24 weeks in comparison with the level at baseline. In these studies, ${ }^{24-28}$ the differences in the characteristics of the studied patients and the control drug used may have contributed to the different results. Furthermore, the study periods in these studies were within 26 weeks, and there was no study with a study period $>26$ weeks.

The REASON Trial has some unique characteristics, and they are as follows: (1) comparisons between two different kinds of DPP-4 inhibitors (SITA and ANA), (2) the study period was 52 weeks, which is longer than that in other studies, ${ }^{24-28}$ and (3) all the examined patients took some type of statin and had a history of atherosclerotic vascular lesions. In this present sub-analysis, the levels of the inflammatory parameters hs-CRP, WBC, and IL-6 were not significantly different between baseline and final 
Table I Patients Characteristics

\begin{tabular}{|c|c|c|c|c|}
\hline & & All Patients $(n=353)$ & ANA $(n=177)$ & SITA $(n=176)$ \\
\hline \multicolumn{2}{|l|}{ Age (yrs) } & $68(10)$ & $68(10)$ & $68(9)$ \\
\hline \multicolumn{2}{|l|}{ Men (\%) } & $214(6 I)$ & $110(62)$ & $104(59)$ \\
\hline \multicolumn{2}{|c|}{ Body mass index } & $26.17(3.79)$ & $26.49(4.04)$ & $25.85(3.50)$ \\
\hline \multirow[t]{2}{*}{ Smoking } & Current (\%) & $54(15)$ & $30(17)$ & $24(14)$ \\
\hline & Past (\%) & $|4|(40)$ & $62(35)$ & $79(45)$ \\
\hline \multicolumn{2}{|c|}{ Hypertension (\%) } & $270(76)$ & 137 (77) & $133(76)$ \\
\hline \multicolumn{2}{|c|}{ Established CAD (\%) } & $159(45)$ & $80(45)$ & $79(45)$ \\
\hline \multicolumn{2}{|c|}{ Taking moderate statin (\%) } & $278(79)$ & $142(80)$ & $136(77)$ \\
\hline \multicolumn{2}{|c|}{ Taking aspirin (\%) } & $153(43)$ & $84(47)$ & $69(39)$ \\
\hline \multicolumn{2}{|c|}{ Taking thiazoridine (\%) } & $56(16)$ & $25(14)$ & $31(18)$ \\
\hline \multicolumn{2}{|c|}{ Taking SGLT2-inhibitor (\%) } & $56(16)$ & $28(16)$ & $28(16)$ \\
\hline \multicolumn{2}{|c|}{ Taking previous DDP-4 inhibitor (\%) } & $290(82)$ & $145(82)$ & $145(82)$ \\
\hline \multicolumn{2}{|c|}{ Total cholesterol (mg/dL) } & $185(169-204)$ & $188(|7|-207)$ & $183(167-199)$ \\
\hline \multicolumn{2}{|c|}{ Triglyceride (mg/dL) } & $120(86-167)$ & $129(95-186)$ & $114(80-159)$ \\
\hline \multicolumn{2}{|c|}{ HDL-cholesterol (mg/dL) } & $52(45-6 I)$ & $52(45-60)$ & $53(45-62)$ \\
\hline \multicolumn{2}{|c|}{ LDL-cholesterol (mg/dL) } & $108(96-122)$ & $112(97-123)$ & $106(94-121)$ \\
\hline \multicolumn{2}{|c|}{ Fasting blood sugar (mg/dL) } & $133(116-118)$ & $136(115-116)$ & $128(117-153)$ \\
\hline \multicolumn{2}{|c|}{ Hemoglobin AIC (\%) } & $6.9(6.4-7.4)$ & $7.0(6.4-7.6)$ & $6.8(6.4-7.3)$ \\
\hline \multicolumn{2}{|c|}{ WBC $\left(/ \mathrm{mm}^{3}\right)$} & $6100(5200-7200)$ & $6365(5325-7500)$ & $5900(4900-7000)$ \\
\hline \multicolumn{2}{|c|}{$\mathrm{hs}-\mathrm{CRP}(\mathrm{mg} / \mathrm{L})$} & $0.658(0.316-1.475)$ & $0.675(0.360-1.450)$ & $0.608(0.307-1.515)$ \\
\hline \multicolumn{2}{|c|}{ IL6 (ng/mL) } & $2.1(1.5-3.2)$ & $2.2(1.4-3.4)$ & $2.1(1.5-2.8)$ \\
\hline
\end{tabular}

Note: Variables are presented as mean (standard deviations) or medians with interquartile ranges.

Abbreviations: ANA, anagliptin; CAD, coronary artery disease; DDP-4, dipeptidyl peptidase-4; HDL, high-density lipoprotein; hs-CRP, high-sensitive C-reactive protein; IL, interleukin; LDL, low-density lipoprotein; SITA, sitagliptin; SGLT-2, sodium glucose co-transporter 2; WBC, white blood cells.

Table 2 Changes in Inflammatory Markers

\begin{tabular}{|c|c|c|c|c|c|c|c|}
\hline & \multicolumn{3}{|l|}{ ANA } & \multicolumn{3}{|l|}{ SITA } & \multirow{3}{*}{$\frac{P \text { value }}{\text { (2 Groups) }}$} \\
\hline & \multirow[t]{2}{*}{ Baseline } & \multirow[t]{2}{*}{$\Delta$ (52W-Baseline) } & \multirow{2}{*}{$\begin{array}{l}\text { P value } \\
\begin{array}{l}\text { (baseline vs } \\
52 \mathrm{~W})\end{array}\end{array}$} & \multirow[t]{2}{*}{ Baseline } & \multirow[t]{2}{*}{$\Delta$ (52W-Baseline) } & \multirow{2}{*}{$\begin{array}{l}\text { P value } \\
\text { (baseline vs } \\
52-W)\end{array}$} & \\
\hline & & & & & & & \\
\hline hs-CRP (mg/L) & $\begin{array}{l}0.675(0.360,1.450) \\
(\mathrm{n}=152)\end{array}$ & $0.495(1.201)$ & 0.61 & $\begin{array}{l}0.608(0.308,1.515) \\
(n=160)\end{array}$ & $-0.252(3.867)$ & 0.41 & 0.46 \\
\hline WBC $\left(/ \mathrm{mm}^{3}\right)$ & $\begin{array}{l}6365(5325,7500) \\
(n=169)\end{array}$ & $-49.42(1339.56)$ & 0.67 & $\begin{array}{l}5900(4900,7000) \\
(n=160)\end{array}$ & $157.63(1482.59)$ & 0.21 & 0.22 \\
\hline IL-6 (pg/dL) & $\begin{array}{l}2.2(1,4,3.4) \\
(n=152)\end{array}$ & $0.694079(5.6774 I)$ & 0.13 & $\begin{array}{l}2.1(1.5,2.8) \\
(n=170)\end{array}$ & $0.151250(3.22338)$ & 0.55 & 0.3 \\
\hline
\end{tabular}

Note: Variables are presented as medians with interquartile ranges or mean (standard deviations).

Abbreviations: ANA, anagliptin; hs-CRP, high-sensitive C-reactive protein; IL, interleukin; No., number; SITA, sitagliptin; WBC, white blood cells.

follow-up in each arm. Moreover, we evaluated the relationship between the change in the hs-CRP level and some clinical factors. It has been well known that many clinical factors influence the hs-CRP level, ${ }^{33}$ and we adopted the factors, such as baseline hs-CRP level, use of a moderateintensity statin, presence of established CAD, other than the absence of prior DPP-4 inhibitor use, which was the important factor for the present subanalyses; none of them affect the change in hs-CRP level. Based on our results, the two kinds of DPP-4 inhibitors, ANA and SITA, that were used in the REASON Trial had no significant effect on the inflammatory markers after 52 weeks of treatment. Such findings support clinical studies that have shown a lack of favorable effects on cardiovascular outcomes from adding DPP-4 inhibitors to usual care in diabetic patients with established cardiovascular diseases. ${ }^{7-10}$ 


\begin{tabular}{|c|c|c|c|c|c|c|c|c|}
\hline & $\mathbf{n}$ & ANA & SITA & ANA better & SITA better & Estimate & $95 \% \mathrm{CI}$ & Interaction $\mathbf{P}$ \\
\hline \multicolumn{9}{|c|}{ Baseline level of hs-CRP } \\
\hline$\geqq 0.658$ & 154 & -0.438 & -1.021 & - & & 0.583 & $-2.584-3.750$ & 0.804 \\
\hline$<0.658$ & 158 & 1.505 & 4.264 & & -1 & 1.079 & $-1.304-3.462$ & \\
\hline \multicolumn{9}{|c|}{ Taking a moderate-strong statin } \\
\hline Yes & 243 & 0.637 & -0.262 & & & 0.898 & $-1.543-3.340$ & 0.756 \\
\hline No & 69 & -0.080 & -0.222 & & - & 0.149 & $-2.275-2.560$ & \\
\hline \multicolumn{9}{|c|}{ Presence of established CAD } \\
\hline Yes & 140 & -0.013 & -0.825 & $\vdash$ & & 0.817 & $-3.202-4.825$ & 0.961 \\
\hline No & 172 & 0.918 & 0.205 & & 7 & 0.714 & $-0.783-2.210$ & \\
\hline \multicolumn{9}{|c|}{ Taking a previous DPP-4 inhibitor } \\
\hline Yes & 254 & 0.635 & -0.182 & & & 0.817 & $-1.587-3.221$ & 0.869 \\
\hline No & 58 & -0.150 & -0.543 & & & 0.393 & $-0.872-1.656$ & \\
\hline & & & & $\begin{array}{rrrr} & -1 & 1 & \\
-4 & -3 & -2 & -1\end{array}$ & $\begin{array}{l}1 \\
2\end{array}$ & & & \\
\hline
\end{tabular}

Figure 2 Subgroup analyses of the difference in the level of high-sensitivity C-reactive protein (hs-CRP).

Abbreviations: ANA, anagliptin; CAD, coronary artery disease; DPP-4, dipeptidyl peptidase-4; SITA, sitagliptin.

Several explanations can be provided for the present results showing no significant anti-inflammatory effect of the two kinds of DPP-4 inhibitors. First, the doses of the DPP-4 inhibitors adopted in the present study may be insufficient to reduce the inflammatory markers. Most of the studied patients received the standard dose of DPP-4 inhibitors. Furthermore, in general, it has been shown that a reduction in LDL-C can cause a reduction in hs-CRP level. ${ }^{16}$ The main result of the REASON Trial was the finding that ANA was deemed superior to SITA in lowering LDL-C by the estimated treatment difference of 4.5 $\mathrm{mg} / \mathrm{dL}$; however, such a small difference in LDL-C was also found insufficient to cause a significant change in hsCRP level. Second, all of the patients studied had taken statins, which have an anti-inflammatory effect, ${ }^{34}$ and the baseline level of CRP was low in both study arms. These findings suggest that many of the studied patients had a stable inflammatory state, showing no significant change in inflammatory markers. Third, the biological counterreaction to the inhibitions of DPP-4 may occur within 52 weeks. In a clinical study using ANA, ${ }^{27}$ the level of CRP at 12 weeks was significantly reduced; however, the level at 24 weeks was not reduced when compared with the level at baseline. This may indicate that the inhibition of DPP-4 and anti-inflammatory reaction was present in the acute phase; however, a biological counterreaction to this reaction may occur in the late phase, further leading to the nonsignificant reduction in the levels of the inflammatory markers. Lastly, the study period of the REASON Trial was 52 weeks, and it is possible that external factors may have affected our results. Actually, it has been reported that there is a seasonal variation in CRP levels and that the presence of passive smoking may affect the level of CRP. $^{35}$ These factors may counter the anti-inflammatory effect of DPP-4 inhibitors in the present sub-analysis of the REASON Trial.

There were several limitations to the present study. First, many of the studied patients had taken some kind of DPP-4 inhibitor before the randomization (82\%); thus, there was a small group of studied patients who had not taken DPP-4 inhibitors (DPP-4 inhibitors naïve), in whom it might be expected that starting to take a DPP-4 inhibitor could have an influence on the levels of inflammatory markers. Second, to analyze the effect of DPP-4 inhibitors on inflammatory markers as the surrogate marker of cardiovascular outcomes not as an acute inflammatory marker, the period of 52 weeks may be too short. Thus, longer follow-up periods may be needed. Third, the setting of the numbers of studied patients in the REASON Trial was not originally aimed for these sub-analyses. Fourth, the REASON Trial did not employ a control group; thus, the natural course of inflammatory markers for 52 weeks was 
unclear. Finally, many factors are reported to influence the hs-CRP level, ${ }^{33}$ and we adopted several factors, such as baseline hs-CRP level, use of a moderate-intensity statin and presence of established CAD, because we had considered the presentative factors influencing hs-CRP level. However, we did not evaluate the validity of all the factors influencing the hs-CRP level.

\section{Conclusions}

In the sub-analysis of the REASON Trial, the two different types of DPP-4 inhibitors, ANA and SITA, were determined to have no significant effect on the inflammatory markers in patients with diabetes and established cardiovascular lesions who were taking any kind of statin.

\section{Abbreviations}

ANA, anagliptin; CAD, coronary artery disease; DM, diabetes mellitus; DPP-4, dipeptidyl peptidase-4; GLP-1, glucagon-like peptide-1; hs-CRP, high-sensitivity C-reactive protein; IL-6, interleukin-6; LDL-C, low-density lipoprotein cholesterol; REASON Trial, Randomized Evaluation of Anagliptin and Sitagliptin in reducing low-density lipoprotein cholesterol in diabetes Trial; SITA, sitagliptin; WBC, white blood cells.

\section{Data Sharing Statement}

The data sets analysed during the current study are available from the corresponding author on reasonable request hiroteraga71@gmail.com.

\section{Ethical Approval}

This study was conducted in accordance with the Declaration of Helsinki and the Ethical Guidelines for Medical and Health Research Involving Human Subjects in Japan. The institutional review boards of the University of the Ryukyus (No. 731) and each participating center gave their approval for this study. This trial was registered on ClinicalTrials.gov (NCT02330406).

\section{Informed Consent}

All patients or their legally authorized representatives provided written informed consent prior to randomization.

\section{Acknowledgments}

The authors are indebted to Ms. Makiko Ohtorii, Ms. Ai Sunagawa, Ms. Sachiko Kitamura, Ms. Kaori Yamamoto, Ms. Hirono Saito, and Ms. Saeko Nagano for their role in data management and Ms. Takako Okumura and for their project management. Dr Osamu Arasaki is now affiliated with the Department of Cardiology, Yuuai Medical Center, Tomigusuku, Okinawa, Japan. Dr Takashi Nomiyama is now affiliated with the Department of Diabetes, Metabolism and Endocrinology, International University of Health and Welfare Ichikawa Hospital, Ichikawa, Japan.

\section{Funding}

This study was supported by Kowa, and the representative of Kowa participated in the study concept and reviewing of the final manuscript; however, the study design, operation, data collection, statistical analyses, and drafting the manuscript were solely conducted by the academic authors.

\section{Disclosure}

Dr. Hiroki Teragawa reports lecturer fees from Daichi Sankyo, Mitsubishi Tanabe, Bayer, Kowa and Nihon Medi-Physics. Dr. Takeshi Morimoto reports lecturer fees from AbbVie, AstraZeneca, Daiichi Sankyo, Kyorin, Mitsubishi Tanabe, Pfizer, and Bayer; manuscript fees from Pfizer; advisory boards for Asahi Kasei, Boston Scientific, Bristol-Myers Squibb, and Sanofi. Dr. Yuichi Fujii declares no conflict of interest. Dr. Tomohiro Ueda declares no conflict of interest. Dr. Mio Sakuma reports advisory board for Enomoto Pharmaceutical. Dr. Michio Shimabukuro reports research grants from AstraZeneca, Ono, and Sanwa Kagaku Kenkyusho; non-purpose research grants from Astellas, AstraZeneca, Bayer, Boehringer Ingelheim, Chugai, Eli Lilly, Kowa, Mitsubishi Tanabe, MSD, Novo Nordisk, Ono, Taisho Toyama, and Takeda; lecturer fees from Astellas, AstraZeneca, Bayer, Boehringer Ingelheim, Chugai, Eli Lilly, Kowa, Mitsubishi Tanabe, Mochida, MSD, Novo Nordisk, Ono, Taisho Toyama, and Takeda; advisory board for Novo Nordisk; and in sponsored office from Boehringer Ingelheim. Dr. Osamu Arasaki reports lecturer fees from Abbott, Astellas, Boehringer Ingelheim, Medtronic, and St. Jude Medical. Dr. Koichi Node reports research grants from Abbott, Actelion, Air Water, Asahi Kasei, Astellas, Bayer, Boehringer Ingelheim, GlaxoSmithKline, Mebix, Mitsubishi Tanabe, and Teijin; non-purpose research grants from Abbott, Astellas, AstraZeneca, Boehringer Ingelheim, Bristol-Myers Squibb, Daiichi Sankyo, Eisai, Eli Lilly, Japan Lifeline, Kissei, Medtronic, Mitsubishi Tanabe, MSD, Novartis, Novo Nordisk, Ono, Otsuka, Pfizer, Sanofi, Sumitomo Dainippon, Takeda, and Teijin; lecturer fees from Abbott, Actelion, AnGes, Astellas, Astellas Amgen Bio Pharma, AstraZeneca, Bayer, Boehringer Ingelheim, Bristol-Myers Squibb, Daiichi 
Sankyo, Edwards Lifesciences, Eisai, Eli Lilly, Fukuda Denshi, Kowa, Kyowa Hakko Kirin, Medtronic, Mitsubishi Tanabe, Mochida, MSD, Nippon Shinyaku, Novartis, Ono, Otsuka, Pfizer, Roche Diagnostics, Sanofi, Sanwa Kagaku Kenkyusho, Sumitomo Dainippon, Taisho Toyama, Takeda, Teijin, and Toa Eiyo; manuscript fee from Takeda; advisory boards for Astellas, AstraZeneca, Boehringer Ingelheim, Eli Lilly, Mitsubishi Tanabe, MSD, Novo Nordisk, Pfizer, and Takeda. Dr. Takashi Nomiyama reports research grants from Eli Lilly, Mitsubishi Tanabe, MSD, and Novartis; lecturer's fees from Arkray, Astellas, AstraZeneca, Boehringer Ingelheim, Eli Lilly, Johnson \& Johnson, Mitsubishi Tanabe, MSD, Novartis, Novo Nordisk, Ono, Sanofi, Sanwa Kagaku Kenkyusho, Sumitomo Dainippon, Taisho Toyama, Takeda, and Terumo. Dr. Shinichiro Ueda reports research grants from Bristol-Myers Squibb, and Kowa; non-purpose research grants from Bristol-Myers Squibb, Chugai, MSD, Pfizer, and Takeda; lecturer fees from Boehringer Ingelheim, and MSD. The authors report no other conflicts of interest in this work.

\section{References}

1. Beckman JA, Creager MA, Libby P. Diabetes and atherosclerosis: epidemiology, pathophysiology, and management. JAMA. 2002;287 (19):2570-2581. doi:10.1001/jama.287.19.2570

2. Cosentino F, Grant PJ, Aboyans V, et al. 2019 ESC guidelines on diabetes, pre-diabetes, and cardiovascular diseases developed in collaboration with the EASD. Eur Heart J. 2019. doi:10.1093/eurheartj/ ehz486

3. Ninomiya T. Japanese legacy cohort studies: the Hisayama study. $J$ Epidemiol. 2018;28(11):444-451. doi:10.2188/jea.JE20180150

4. Gaede P, Vedel P, Larsen N, et al. Multifactorial intervention and cardiovascular disease in patients with type 2 diabetes. $N$ Engl J Med. 2003;348(5):383-393. doi:10.1056/NEJMoa021778

5. Davies MJ, D’Alessio DA, Fradkin J, et al. Management of hyperglycemia in type 2 diabetes, 2018. A consensus report by the American Diabetes Association (ADA) and the European Association for the Study of Diabetes (EASD). Diabetes Care. 2018;41(12):2669-2701. doi:10.2337/dci18-0033

6. Drucker DJ. The biology of incretin hormones. Cell Metab. 2006;3 (3):153-165. doi:10.1016/j.cmet.2006.01.004

7. Scirica BM, Bhatt DL, Braunwald E, et al. Saxagliptin and cardiovascular outcomes in patients with type 2 diabetes mellitus. $N$ Engl J Med. 2013;369(14):1317-1326. doi:10.1056/NEJMoa1307684

8. White WB, Cannon CP, Heller SR, et al. Alogliptin after acute coronary syndrome in patients with type 2 diabetes. $N$ Engl J Med. 2013;369(14):1327-1335. doi:10.1056/NEJMoa1305889

9. Green JB, Bethel MA, Armstrong PW, et al. Effect of sitagliptin on cardiovascular outcomes in type 2 diabetes. $N$ Engl J Med. 2015;373 (3):232-242. doi:10.1056/NEJMoa1501352

10. Rosenstock J, Perkovic V, Johansen OE, et al. Effect of linagliptin vs placebo on major cardiovascular events in adults with type 2 diabetes and high cardiovascular and renal risk: the CARMELINA randomized clinical trial. JAMA. 2019;321(1):69-79. doi:10.1001/ jama.2018.18269

11. Ross R, Epstein FH. Atherosclerosis-an inflammatory disease. $N$ Engl J Med. 1999;340(2):115-126. doi:10.1056/NEJM1999011 43400207
12. Ridker PM, Danielson E, Fonseca FA, et al. Rosuvastatin to prevent vascular events in men and women with elevated C-reactive protein. $N$ Engl J Med. 2008;359(21):2195-2207. doi:10.1056/NEJMoa 0807646

13. Ridker PM, Libby P, MacFadyen JG, et al. Modulation of the interleukin-6 signalling pathway and incidence rates of atherosclerotic events and all-cause mortality: analyses from the Canakinumab Anti-Inflammatory Thrombosis Outcomes Study (CANTOS). Eur Heart J. 2018;39(38):3499-3507. doi:10.1093/eurheartj/ehy310

14. Tomovic K, Lazarevic J, Kocic G, et al. Mechanisms and pathways of anti-inflammatory activity of DPP-4 inhibitors in cardiovascular and renal protection. Med Res Rev. 2019;39(1):404-422. doi:10.1002/ med.21513

15. Morimoto T, Sakuma I, Sakuma M, et al. Randomized evaluation of anagliptin vs sitagliptin on low-density lipoproteiN cholesterol in diabetes (REASON) trial: a 52-week, open-label, randomized clinical trial. Sci Rep. 2019;9(1):8537. doi:10.1038/s41598-019-44885-x

16. Ridker PM, Danielson E, Fonseca FA, et al. Reduction in C-reactive protein and LDL cholesterol and cardiovascular event rates after initiation of rosuvastatin: a prospective study of the JUPITER trial. Lancet. 2009;373(9670):1175-1182. doi:10.1016/S0140-6736(09)60447-5

17. Ueda S, Shimabukuro M, Arasaki O, et al. Effect of anagliptin and sitagliptin on low-density lipoprotein cholesterol in type 2 diabetic patients with dyslipidemia and cardiovascular risk: rationale and study design of the REASON trial. Cardiovasc Drugs Ther. 2018;32(1):73-80. doi:10.1007/s10557-018-6776-z

18. Stone NJ, Robinson JG, Lichtenstein A. The new cholesterol treatment guidelines. $N$ Engl J Med. 2014;370(20):1957. doi:10.1056/ NEJMc1403438

19. Hanefeld M, Marx N, Pfutzner A, et al. Anti-inflammatory effects of pioglitazone and/or simvastatin in high cardiovascular risk patients with elevated high sensitivity C-reactive protein: the PIOSTAT study. J Am Coll Cardiol. 2007;49(3):290-297. doi:10.1016/j.jacc.2006.08.054

20. Zhao Y, He X, Huang C, et al. The impacts of thiazolidinediones on circulating C-reactive protein levels in different diseases: a metaanalysis. Diabetes Res Clin Pract. 2010;90(3):279-287. doi:10.1016/j.diabres.2010.09.011

21. Mazidi M, Karimi E, Rezaie P, et al. Treatment with GLP1 receptor agonists reduce serum CRP concentrations in patients with type 2 diabetes mellitus: a systematic review and meta-analysis of randomized controlled trials. J Diabetes Complications. 2017;31(7):12371242. doi:10.1016/j.jdiacomp.2016.05.022

22. Lee YS, Jun HS. Anti-inflammatory effects of GLP-1-based therapies beyond glucose control. Mediators Inflamm. 2016;2016:3094642. doi:10.1155/2016/3094642

23. Garvey WT, Van Gaal L, Leiter LA, et al. Effects of canagliflozin versus glimepiride on adipokines and inflammatory biomarkers in type 2 diabetes. Metabolism. 2018;85:32-37. doi:10.1016/j. metabol.2018.02.002

24. Satoh-Asahara N, Sasaki Y, Wada H, et al. A dipeptidyl peptidase-4 inhibitor, sitagliptin, exerts anti-inflammatory effects in type 2 diabetic patients. Metabolism. 2013;62(3):347-351. doi:10.1016/j. metabol.2012.09.004

25. Nakamura K, Oe H, Kihara H, et al. DPP-4 inhibitor and alphaglucosidase inhibitor equally improve endothelial function in patients with type 2 diabetes: EDGE study. Cardiovasc Diabetol. 2014;13:110. doi:10.1186/s12933-014-0110-2

26. Tremblay AJ, Lamarche B, Deacon CF, et al. Effects of sitagliptin therapy on markers of low-grade inflammation and cell adhesion molecules in patients with type 2 diabetes. Metabolism. 2014;63 (9):1141-1148. doi:10.1016/j.metabol.2014.06.004

27. Kakuda H, Kobayashi J, Kakuda M, et al. The effect of anagliptin treatment on glucose metabolism and lipid metabolism, and oxidative stress in fasting and postprandial states using a test meal in Japanese men with type 2 diabetes. Endocrine. 2015;48(3):1005-1009. doi:10.1007/s12020-014-0376-x 
28. Nomoto H, Miyoshi H, Furumoto T, et al. A randomized controlled trial comparing the effects of sitagliptin and glimepiride on endothelial function and metabolic parameters: sapporo athero-incretin study 1 (SAIS1). PLoS One. 2016;11(10):e0164255. doi:10.1371/journal. pone. 0164255

29. Lovshin JA, Rajasekeran H, Lytvyn Y, et al. Dipeptidyl peptidase 4 inhibition stimulates distal tubular natriuresis and increases in circulating SDF-1alpha(1-67) in patients with type 2 diabetes. Diabetes Care. 2017;40(8):1073-1081. doi:10.2337/dc17-0061

30. Zhong J, Rao X, Deiuliis J, et al. A potential role for dendritic cell/ macrophage-expressing DPP4 in obesity-induced visceral inflammation. Diabetes. 2013;62(1):149-157. doi:10.2337/db12-0230

31. Shi S, Koya D, Kanasaki K. Dipeptidyl peptidase-4 and kidney fibrosis in diabetes. Fibrogenesis Tissue Repair. 2016;9:1. doi:10.1186/s13069-016-0038-0

32. Romacho T, Vallejo S, Villalobos LA, et al. Soluble dipeptidyl peptidase-4 induces microvascular endothelial dysfunction through proteinase-activated receptor- 2 and thromboxane A2 release. J Hypertens. 2016;34(5):869-876. doi:10.1097/HJH.0000000000000886
33. Pearson TA, Mensah GA, Alexander RW, et al. Markers of inflammation and cardiovascular disease: application to clinical and public health practice: a statement for healthcare professionals from the Centers for Disease Control and Prevention and the American Heart Association. Circulation. 2003;107(3):499-511. doi:10.1161/01. cir.0000052939.59093.45

34. Albert MA, Danielson E, Rifai N, et al. Effect of statin therapy on C-reactive protein levels: the pravastatin inflammation/CRP evaluation (PRINCE): a randomized trial and cohort study. JAMA. 2001;286 (1):64-70. doi:10.1001/jama.286.1.64

35. Mustonen K, Keski-Nisula L, Vaarala O, et al. Few associations between high-sensitivity $\mathrm{C}$-reactive protein and environmental factors in 4.5-year-old children. Pediatr Allergy Immunol. 2012;23(6):522528. doi:10.1111/j.1399-3038.2012.01306.x

\section{Publish your work in this journal}

Diabetes, Metabolic Syndrome and Obesity: Targets and Therapy is an international, peer-reviewed open-access journal committed to the rapid publication of the latest laboratory and clinical findings in the fields of diabetes, metabolic syndrome and obesity research. Original research, review, case reports, hypothesis formation, expert opinion and commentaries are all considered for publication. The manuscript management system is completely online and includes a very quick and fair peer-review system, which is all easy to use. Visit http://www.dovepress.com/testimonials.php to read real quotes from published authors. 